Initial Blackbeard Power Survey Results

Tim Murphy

Jim Devenport

Dan Holden
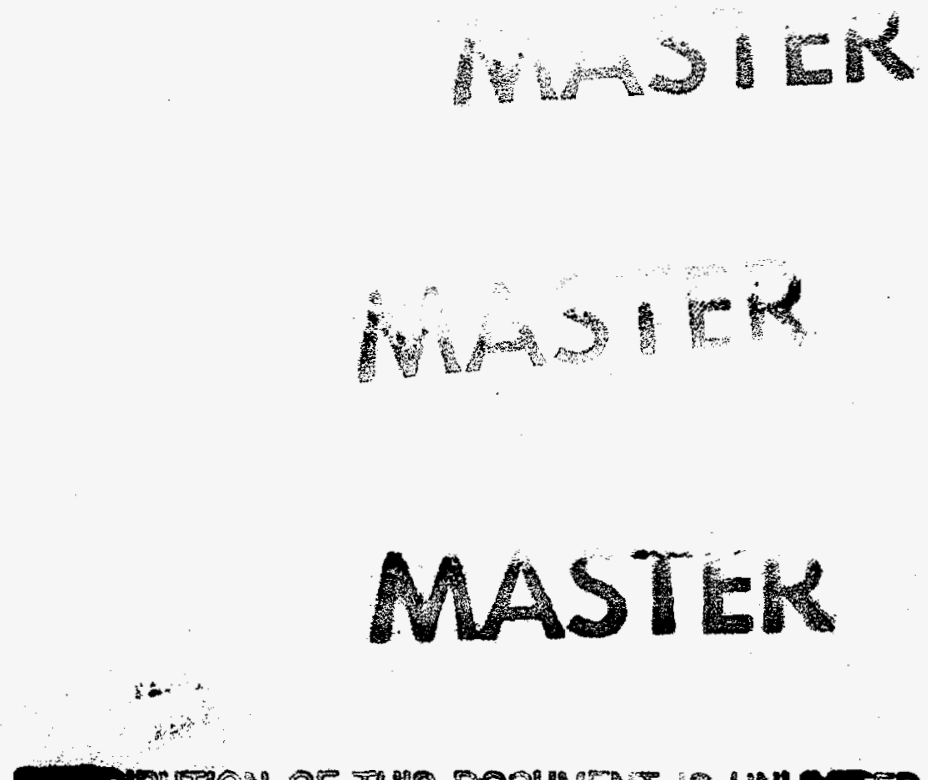

MUTHCN OF THS DOCURAENT IS UNLNTED , the 


\section{DISCLAMMER}

Portions of this document may be illegible in electronic image products. Images are produced from the best available original document. 


\title{
Initial Blackbeard Power Survey Results
}

\author{
by \\ Tim Murphy, Jim Devenport, \& Dan Holden
}

\begin{abstract}
The Blackbeard broadband VHF radio receiver is in low-earth orbit aboard the ALEXIS satellite. The receiver has been used to measure the transmitted power in four VHF bands (55.2-75.8, 28.0-94.8, 132.3-152.2, and 107.7-166.0 $\mathrm{MHz}$ ) over quiet and noisy parts of the earth. We present the results of the survey and disucss their implications. We find that there are remote ocean areas over which the observed spectrum is largely free of man-made interference, but that the spectrum over most of the earth is dominated by broadcast VHF signals. The signal characteristics observed over a given area are quite constant when observed at different times of day and at intervals of several weeks to months. It appears that in many cases the bulk of the signal power is coming from a small number of sources.
\end{abstract}

\section{Purpose of the Observations}

The goal of this experiment was to perform an initial survey of the level of radio frequency (RF) electromagnetic power in the VHF band from 28-166 MHz which will be seen by a broadband radio receiver in low earth orbit. VHF receivers have been flown on satellites for communications and other purposes for many years, but they have generally operated over narrow bands at fixed frequencies, and most of them were not accurately calibrated to measure absolute field strengths. The information available to date is sparse and does not span the full range of VHF frequencies or have complete geographical coverage.

We have both scientific and programmatic reasons for conducting this survey with Blackbeard. From a scientific perspective we expect the information gathered in this experiment to be useful both in and of itself, as information on background noise should be of interest for any orbiting receiver, and as a guide to further work in this area. In addition this work is of immediate programmatic relevance to us at Los Alamos because of the fast on-orbit recording of transient events (FORTE) satellite project. The payload on this satellite will include two wide-band ( $20 \mathrm{MHz})$ and one ultra-wide-band $(90 \mathrm{MHz})$ VHF receivers designed to look for transient events coming from the earth, such as lightning or the recently discovered transionospheric pulse pair (TIPP) events ${ }^{1,2}$. Data on the background is needed by FORTE both to determine the correct ranges for the gain controls of the receivers and for modeling of the signal-detection performance of the system. 


\section{The Blackbeard Receiver}

Blackbeard is a large bandwidth VHF digital radio receiver on the array of low-energy $x$-ray imaging sensors (ALEXIS) satellite. The satellite is in a $750 \times 850$ kilometer, $70^{\circ}$ inclination orbit, spin stabilized, and in a sun-pointing orientation. The receiver is fed by either of two active monopole antennas oriented along the axis of rotation of the spacecraft. Because the satellite points toward the sun, the orientation of the antennas with respect to the earth varies with position in the orbit. In addition to this gross orientation effect, there are azimuthal asymmetries in the monopole antenna pattern due to the solar paddles and other structures on the satellite. Because the satellite is rotating at a spin rate of about one rotation every 45 seconds, the asymmetry in the antenna pattern introduces a periodic variation into the gain of the antenna as seen from a fixed point on the earth.

The Blackbeard receiver can operate in either its low band, with a maximum bandpass of $28.0-94.8 \mathrm{MHz}$, or its high band with a maximum bandpass of $107.7-$ $166.0 \mathrm{MHz}$. Various subbands can be selected within either of these two bands by inserting additional filters, with the desired filter combination selected manually by the operator on the ground. There are 30 possible filter combinations, 15 in the low band and 15 in the high band. After filtering, the bandpass-limited signal is mixed with a $100 \mathrm{MHz}$ local oscillator and then digitized using an 8-bit analog-to-digital converter. The digital levels reported to the ground are converted back to electric field strengths by using the measured transfer function of the receiver.

Blackbeard can be operated in a number of modes for various purposes. The data described in this report was collected using the survey mode, which looks at the power envelope of the signal across the entire subband. When operated in survey mode the total power across the band is sampled at 90 thousand samples/s and recorded in the payload memory in real time, interleaved 3:1 with additional data coming from a separate narrowband channel. The narrowband data was not used in this analysis and will not be discussed further in this report. The maximum length of a Blackbeard data record is 16 Mbytes, spanning a time of about 144 seconds in survey mode. This is just over three full rotations of the spacecraft, allowing us to roughly resolve rotation-dependent effects in the data.

The receiver can also operate in broadband mode, in which it records a time series at 150 million samples/s. This allows us to extract both amplitude and phase information from the signals and to obtain the spectral distribution of power across the band, but it only allows us to record data for a maximum of $1 / 10$ of a second. We have used this mode occasionally in this study to get information on the spectral distribution of the noise.

\section{Design of the Observation Program}

As stated previously, the goal of the experiment was to perform a survey of the ambient VHF power present in the frequency bands accessible to Blackbeard. There are many parameters which should be varied in order to perform a truly 
comprehensive survey, including frequency band, subsatellite point, local time, and time of year. The geometry of the orbit forces a correlation between subsatellite point and local time and there are additional operational constraints that involve the amount of data that can be recorded and downlinked. Because of these limitations, it is not possible to exhaustively examine the variations of the $R F$ power with respect to all of the parameters listed above. Experience with Blackbeard operating in broadband mode indicated that the VHF background has only a weak dependence on time of day and time of year, so we did not attempt to sample these parameters in an organized manner; instead we concentrated on frequency band and subsatellite point.

Blackbeard has 30 possible VHF filter combinations, too many to use all of them in this survey. To get an idea of which bands to concentrate on, we looked at spectra taken in broadband mode. Two spectra for the low band are shown in Figure 1, one taken over the western United States and one taken over Johnston Island in the Pacific Ocean. Both spectra have a nominal pass band of 28.0-94.8 $\mathrm{MHz}$. In looking at these data, we see that over the United States there are a number of commercial transmission bands which dominate the high end of the spectrum. Television stations $2,3,4,5$, and 6 are clearly visible as is the FM band. It appears that the overall background level is higher above about $40 \mathrm{MHz}$; this is probably due to out-of-band power from the transmitters. There are also a few bright sources around 35 and $40 \mathrm{MHz}$. The spectrum over Johnston Island shows traces of the television and FM stations but at much lower levels; these are most likely coming from Hawaii. The levels in both records are fairly close at the low end of the spectrum, which may indicate that the base in this region is essentially the thermal background. The Johnston Island data shows the noise background falling with increasing frequency, as one might expect.

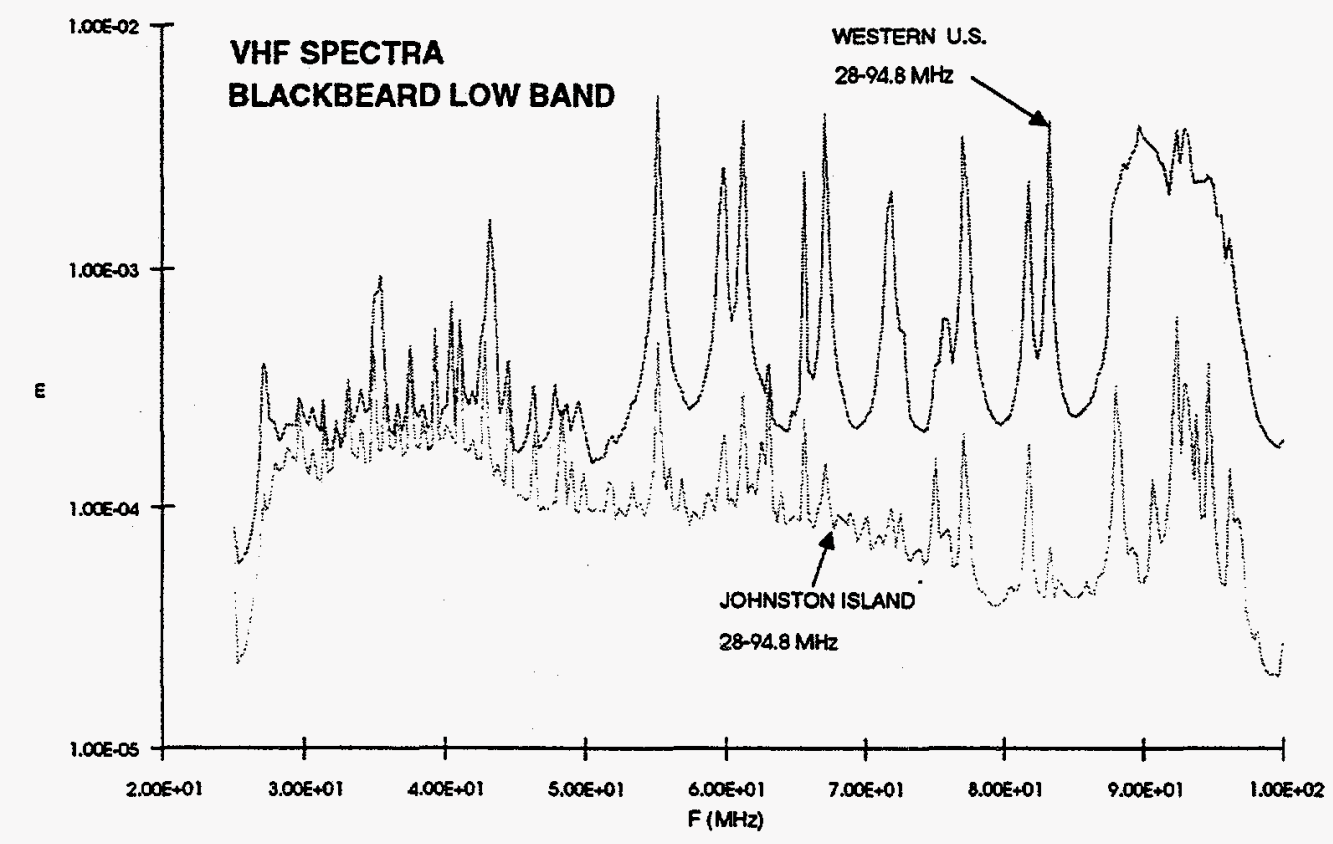

Figure 1 
Two spectra from the high band are shown in Figure 2, one from over the western United States and one from the southeastern Pacific Ocean, near Easter Island. The United States data was taken with a nominal pass band of 117.0-166.0 $\mathrm{MHz}$, while the pass band of 107.7-166.0 MHz was used for the Pacific data. Once again the amplitude of the signal over the United States is higher than for the Pacific. There are no television or FM stations in band for the U.S. data, although we may be seeing some out-of-band response to the FM band below $110 \mathrm{MHz}$. We believe the large peak around $115 \mathrm{MHz}$ is due to aircraft navigation beacons, while the peaks at higher frequencies are coming from intermittent communications traffic such as aircraft or taxi cab dispatchers. The data from the Pacific shows no distinct peaks except for one around $112 \mathrm{MHz}$, which is most likely an airport beacon. The background level appears to be rising with frequency in this band, but we believe this is because the antenna is approaching resonance at the higher frequencies.

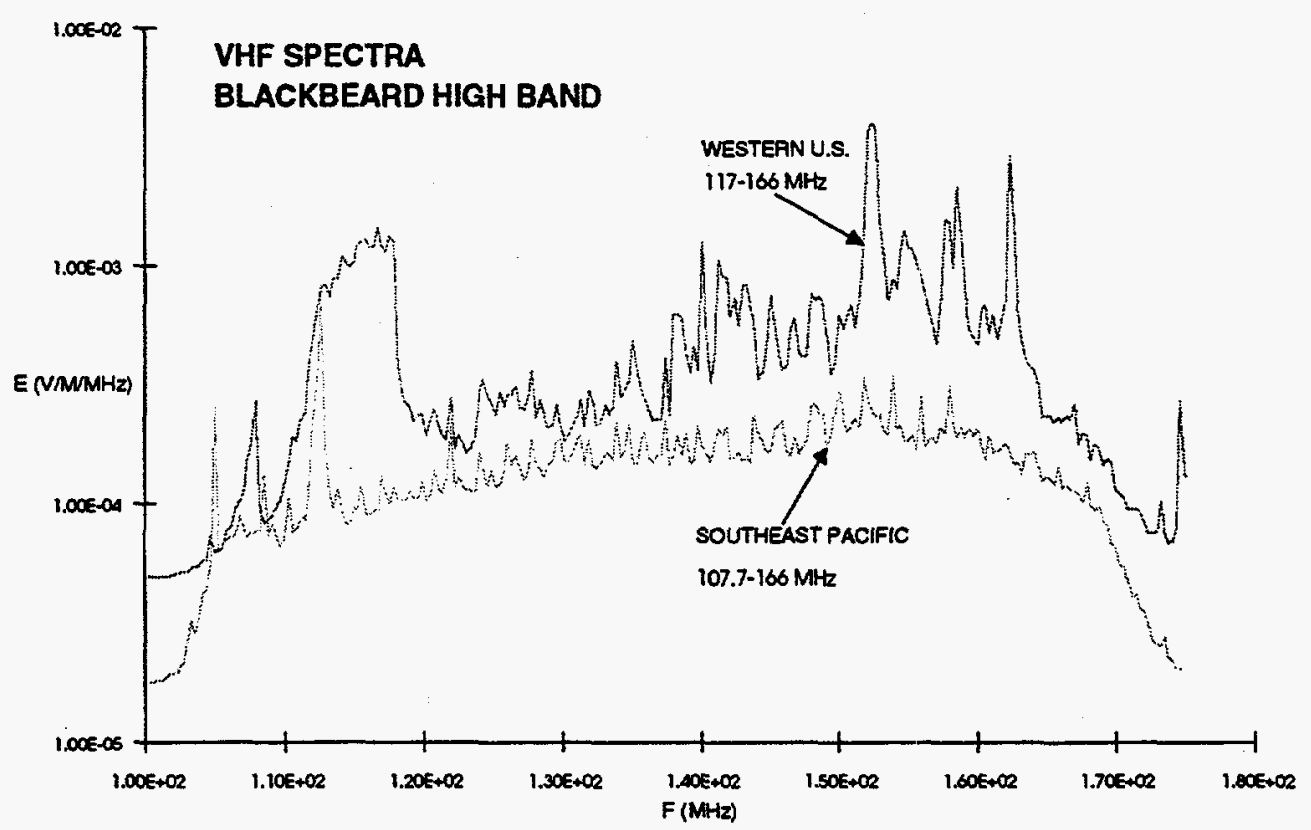

Figure 2

Based on the data in these spectra and the information needed by the FORTE project, we selected four frequency bands out of the 30 possible. The bands and the rationale for their selection are as follows:

- band $\mathrm{A}-55.2-75.8 \mathrm{MHz}$ - the bandwidth was chosen to match that of the FORTE wide-band receivers. This is not the noisiest $20 \mathrm{MHz}$ in the Blackbeard low band, but we expected that it would be the most variable;

- band $\mathrm{B}-28.0-94.8 \mathrm{MHz}$ - this is the maximum bandwidth in the low band mode and is approximately the bandwidth of the FORTE ultra-wide-band receiver; 
- band $\mathrm{C}-132.3-152.2 \mathrm{MHz}$ - this has the right bandwidth for FORTE wideband receivers and is reasonably quiet. It should get a lot of the push-to-talk radios and so be rather variable; and

- band $\mathrm{D}-107.7-166.0 \mathrm{MHz}$ - maximum bandwidth in the high band mode, best fit to FORTE ultra-wide-band.

Rather than try to uniformly sample the earth geographically, we decided to look at selected areas which we expected to be either "noisy" or "quiet". For each of the four frequency bands we took measurements over a noisy area, such as the west coast of the U.S. or northern Europe, and over a quiet area such as the south Pacific or central Africa. In order to see if there was significant day-to-day variation, we tried to take at least four or five measurements over each area, although not necessarily on consecutive days. In addition, we took occasional measurements over other areas, such as Central America or Russia, to see what differences we might find. Data collection began on January 2, 1994, and was completed on June 7. Forty-five measurements were taken in all, over a span of five months.

\section{Data Processing}

The raw data as collected have several systematic effects which would lead to variations in the received power even if the source on the earth were constant. These include changes in the altitude of the satellite, variations in the orientation of the antenna axis with respect to the earth, and variations in the antenna pattern as the satellite rotates. There are significant differences in the responses of the two antennas on the spacecraft, but this did not play a role in these observations because all of the data was collected using the same antenna. We have attempted to account for the altitude variations by performing $1 / R^{2}$ scaling of the data to a uniform altitude of $800 \mathrm{~km}$, so that if $E_{\mathrm{r}}$ is the raw signal and $E_{\mathrm{s}}$ is the scaled signal, we have $E_{s}=E_{r} R^{2} / R_{0}{ }^{2}$, where $R$ is the altitude of the satellite at the time of the measurement and $R_{0}=800 \mathrm{~km}$. This is, strictly speaking, not correct because the scaling factor for a given source depends on the range and will be different for different sources, and we do not know the location of the sources anyhow.

Nevertheless this scaling does dramatically reduce the scatter in the data and so we have applied it. We have not corrected for the antenna axis orientation. The effects due to spacecraft rotation are obvious in the data and will be discussed in the analysis section of this report.

Most of the data records used in this report utilize the full 16 Mbytes available, although several are shorter for various reasons. The satellite moves at about $7 \mathrm{~km} / \mathrm{s}$ while its footprint on the ground is approximately $6000 \mathrm{~km}$ across. Thus if the background source power is fairly constant and the variation is mostly due to the changing field of view, one would expect changes to occur on a time scale of seconds. Because of the large size of the data files and because the sampling rate was far higher than that needed to resolve variations on the one-second time scale of interest, the data was binned into groups of 32768 samples, with a time step of 
about 0.36 seconds. It is these binned data that will be discussed in this report. We also calculated the standard deviation of the binned data to see whether the binning process was causing a significant loss of information. The ratio of the standard deviation to the mean in each bin was typically about $10 \%-15 \%$ over noisy areas and 3\% over quiet areas, indicating that the bin averages are a good indication of the trends in the data. Finally, the binned data were Fourier analyzed to see if fluctuations in the signal were random or occurred at specific frequencies, which brought out satellite rotation effects very clearly. Of course, the original high-time resolution data is available if variations on at finer time scale are of interest.

\section{$\underline{\text { Results }}$}

\section{General :}

The most striking feature of this data is how repeatable the signals are over a given geographical area. As stated previously, we expected there to be little dependence on local time or day of year, and this is confirmed by our results. Typical too-mean-square (RMS) electric field strengths for the four bands used in this experiment are shown in Table 1. The Fourier spectra of most of the data showed a peak at $0.022 \mathrm{~Hz}$, which is the spacecraft rotation frequency. This indicates that an appreciable part of the noise comes from localized sources so that as the antenna pattern rotates these sources move in and out of the lobes. Some of the records did not show this effect, which indicates that the sources of the noise were approximately uniformly distributed.

Table 1. Typical RMS Electric Field Strengths

\begin{tabular}{ccc}
\hline Band & Noisy Area Field Strength & Quiet Area Field Strength \\
\hline A & $3-7 \mathrm{mV} / \mathrm{m}$ & $0.25 \mathrm{mV} / \mathrm{m}$ \\
B & $5-8 \mathrm{mV} / \mathrm{m}$ & $0.6 \mathrm{mV} / \mathrm{m}$ \\
C & $1-2.5 \mathrm{mV} / \mathrm{m}$ & $0.7 \mathrm{mV} / \mathrm{m}$ \\
D & $4-5 \mathrm{mV} / \mathrm{m}$ & $0.8 \mathrm{mV} / \mathrm{m}$ \\
\hline
\end{tabular}

Although our choices of noisy and quiet areas turned out to be more or less correct, there were several interesting features in our results. We had anticipated that the United States would be the noisiest environment, but in fact the fields measured over Europe were generally somewhat higher. It is also rather surprising how quiet it is in the VHF spectrum over Africa. We now turn to discussion of the detailed results for each band. 


\section{Band A : 55.2-75.8 MHz}

We will begin with the $55.2-75.8 \mathrm{MHz}$ channel. We expected this band to be fairly noisy over populated areas because it typically includes several television channels. For example, it includes channels 2, 3, and 4 in the United States; E-3 and E-4 in Northern Europe; R2 in Russia; and 1 and 2 in Australia. Because television video signals are amplitude modulated and also include a strong synchronization pulse, we expected the instantaneous power to be somewhat variable but to have a steady time average.

Twelve records were taken: four over quiet areas and the remaining eight over noisy areas. The data for all twelve records are shown in Figure 3, where the amplitudes have been binned and $1 / \mathrm{R}^{2}$ scaled as discussed above. Details of the time and subsatellite points are given in Table 2. We see that the quiet-area records and the noisy-area records are cleanly separated, and that the noisy-area records themselves fall into several groups. We now turn to a detailed discussion of these records.

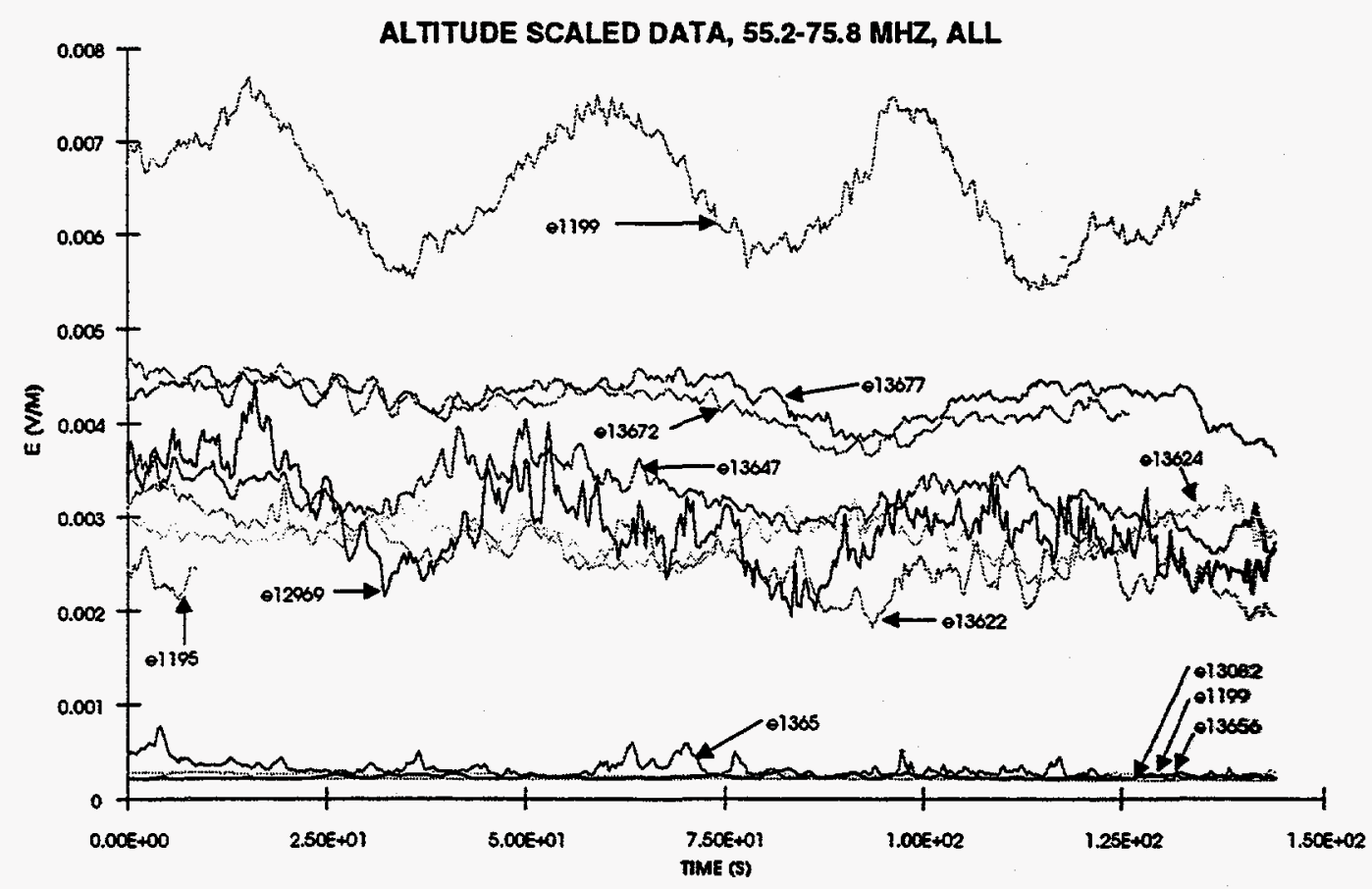

Figure 3

We begin with the quiet-area data, shown separately in Figure 4. The data taken from the southernmost latitude, file 13082 , shows very little variation. The constancy of this signal implies that there are no point sources of VHF, such as transmitters, in the field of view and is what one would expect from thermal noise. This interpretation is supported by the measurements taken over this region in band B, which also show a very flat signal with an amplitude about three times larger than seen here, which is roughly the ratio of the bandwidths. 
Table 2. 55.2-75.8 MHz Data

\begin{tabular}{lrrrrrrl}
\hline File & \multicolumn{1}{c}{ Date } & \multicolumn{1}{c}{ Time(UT) } & Time (loc) & \multicolumn{1}{c}{ Lat. } & Long. & Alt. (km) & Subsatellite Point \\
\hline 13082 & $2 / 19 / 94$ & $19: 29: 49$ & $19: 40: 05$ & -69.91 & 2.57 & 833.88 & Antarctica \\
11994 & $1 / 10 / 94$ & $7: 54: 49$ & $10: 06: 23$ & -12.08 & 32.89 & 832.32 & Mozambique \\
13651 & $5 / 18 / 94$ & $13: 49: 49$ & $18: 49: 35$ & -10.43 & 74.94 & 822.90 & Indian Ocean \\
13656 & $5 / 19 / 94$ & $14: 59: 49$ & $18: 32: 03$ & -7.85 & 53.06 & 823.62 & Madagascar \\
13622 & $5 / 11 / 94$ & $3: 44: 49$ & $19: 42: 08$ & 18.81 & -120.67 & 836.07 & W. of Baja \\
13624 & $5 / 12 / 94$ & $1: 30: 49$ & $19: 10: 44$ & 29.74 & -95.02 & 835.82 & Houston \\
13647 & $5 / 17 / 94$ & $13: 37: 49$ & $7: 56: 56$ & 39.31 & -85.22 & 779.20 & Cincinnati \\
13672 & $5 / 22 / 94$ & $13: 11: 49$ & $15: 55: 03$ & 51.01 & 40.81 & 833.97 & Rostov, Russia \\
12969 & $2 / 16 / 94$ & $9: 56: 49$ & $4: 28: 01$ & 55.16 & -82.20 & 755.21 & Moosonee \\
11958 & $1 / 2 / 94$ & $4: 31: 41$ & $19: 00: 31$ & 56.26 & -142.79 & 753.71 & Gulf of Alaska \\
13677 & $5 / 23 / 94$ & $12: 39: 49$ & $15: 13: 15$ & 56.91 & 38.36 & 832.38 & Moscow \\
11995 & $1 / 10 / 94$ & $20: 14: 49$ & $19: 35: 47$ & 60.61 & -9.76 & 754.60 & Shetlands \\
\hline
\end{tabular}

Two records were taken over the Mozambique-Madagascar area, files 11994 and 13656. These show a slightly higher and more variable signal. The record taken over Mozambique is quite smooth and does not exhibit any systematic variation with spacecraft orientation. The record taken over Madagascar shows much more rapid and regular variations than the one taken over Mozambique, which we attribute to a bright source which is seen in different parts of the antenna pattern as the satellite rotates. This is borne out by a Fourier analysis of the data,

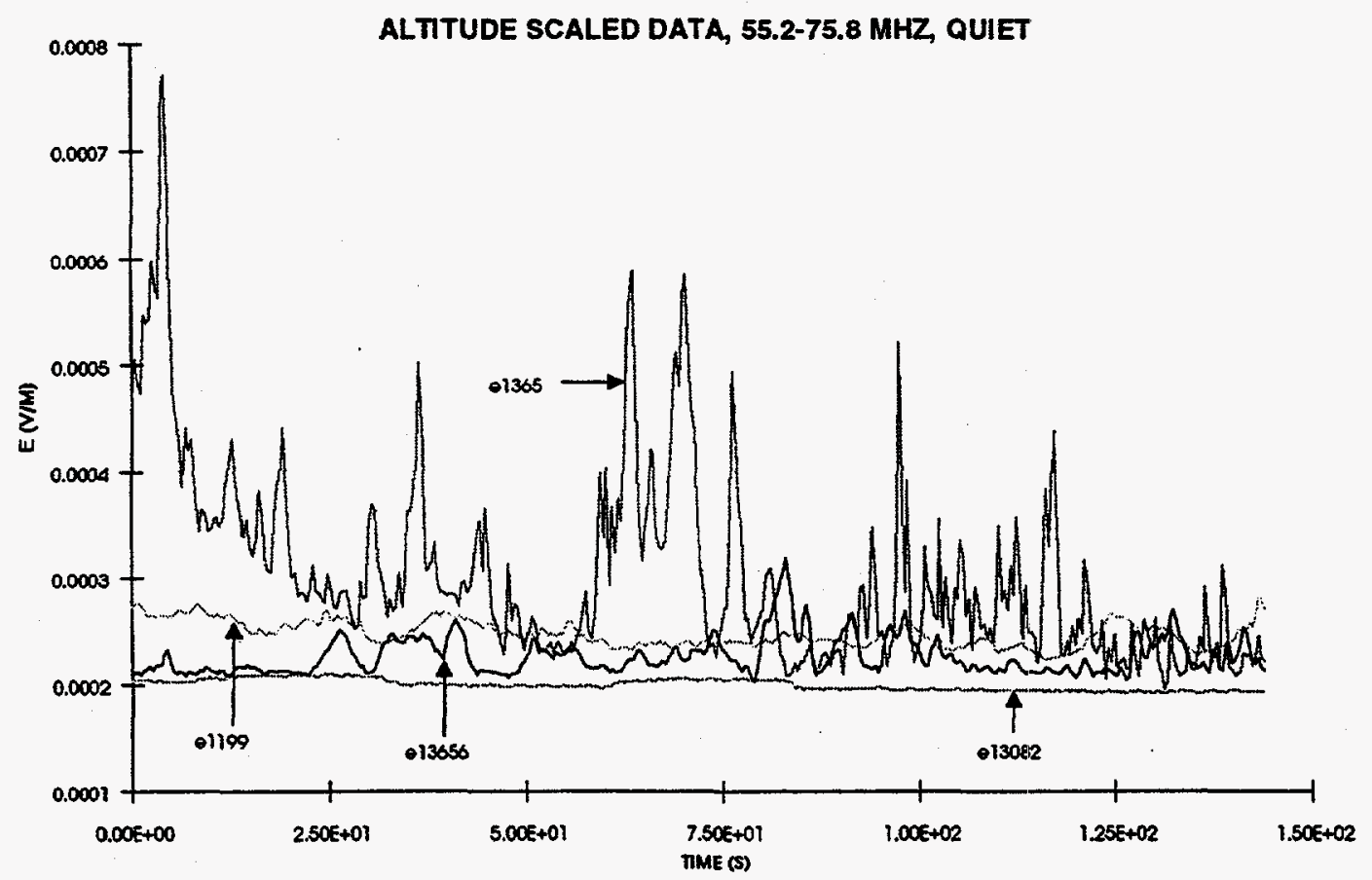

Figure 4 
which shows a clear peak at $0.022 \mathrm{~Hz}$, the spacecraft rotation period. The presence of a well-defined source in one record but not the other may be explained by the fact that some of the Persian Gulf oil states are on the fringe of the field of view for record 13656 but not 11994 .

The last of the quiet records is file 13651 which shows a much stronger and more variable signal than the other three. The field of view for this data does not include any of the continental land masses but does include Diego Garcia, which has a large military base and is a source of significant VHF emanations. The spectrum of this data also shows a strong peak at $0.022 \mathrm{~Hz}$, supporting the idea of a localized source.

Let us turn now to the noisy-area data, shown in Figure 5. This can be broken into three distinct sets. The first is that taken over the United States and Canada, files 11958, 12969, 13622, 13624, and 13647. Each record shows a fair amount of variability, but taken together we see that they overlay each other very well, despite substantial variations in location of the subsatellite point and the time at which the data was collected. File 11958, the record taken over the Gulf of Alaska, may be somewhat lower in amplitude than the others, but it is so short it is hard to tell if that is a significant trend or simply a variation. Surprisingly, all of the files except 13622 show a very strong peak at $0.022 \mathrm{~Hz}$; this indicates the presence of a small number of discrete strong transmitters. One might have expected this effect to be the most pronounced for 13622 , where the subsatellite point is well out to sea and all of the land in the field of view is concentrated in one quadrant, but in fact this is the record with the weakest rotational dependence.

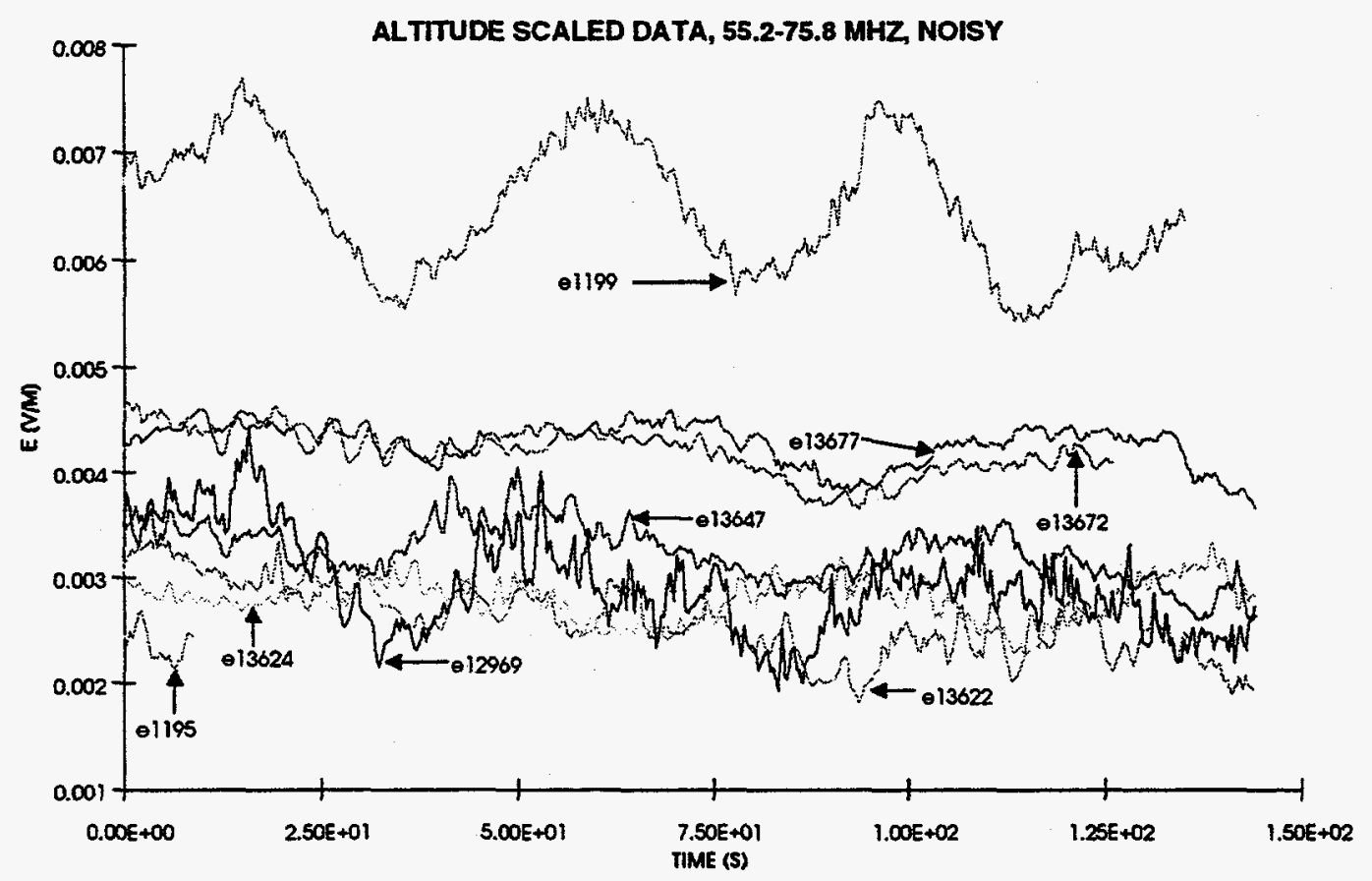

Figure 5 
The second set consists of two records taken over Russia, files 13672 and 13677. The signals here are slightly but noticeably stronger than over North America and are remarkably uniform. Although it is not obvious in the timedomain records, file 13672 does not have a strong $0.022 \mathrm{~Hz}$ component and file 13677 does. We interpret the high amplitude but small variation of the signals to mean that the background consists of a large number of low-powered transmitters.

The third set is the final record in this band, file 11995, taken over the North Sea. This record clearly stands apart from the rest both because of the amplitude of the signal and the very clear 45-second period that can be seen even in the time domain. We interpret this to mean that the data is being dominated by a few powerful transmitters, possibly in the North Sea oil fields.

\section{Band B : 28.0-94.8 MHz}

Next we turn to the data taken in the low band with the full bandwidth available, $28.0-94.8 \mathrm{MHz}$. This encompasses band $\mathrm{A}$, with the transmitters in that band as discussed above, as well as a number of additional commercial transmitting frequencies, which includes other television channels and FM radio. With a greater number of transmitters in band we expected the overall signal level to be higher but less variable than in band $A$. We have a total of ten records in this band: four over quiet areas, five over noisy areas, and one intermediate case. Details of the records are given in Table 3 and the complete set of data is shown in Figure 6. As in band $A$, the data over quiet areas is clearly separated from that over noisy areas, and the noisy data itself shows several distinct groups.

Table 3. 28.0-94.8 MHz Data

\begin{tabular}{rrrrrrrl}
\hline \multicolumn{1}{l}{ File } & \multicolumn{1}{c}{ Date } & Time(UT) & Time (loc) & \multicolumn{1}{c}{ Lat. } & Long. & Alt. (km) & Subsatellite Point \\
\hline 13565 & $4 / 30 / 94$ & $4: 46: 49$ & $6: 54: 49$ & -65.86 & 32.00 & 773.98 & Antarctica \\
13559 & $4 / 29 / 94$ & $3: 39: 49$ & $8: 32: 20$ & -56.61 & 73.13 & 765.79 & McDonald Isl. \\
12029 & $1 / 12 / 94$ & $18: 09: 53$ & $23: 57: 12$ & -54.72 & 86.83 & 796.92 & Indian Ocean \\
12028 & $1 / 12 / 94$ & $3: 30: 54$ & $9: 28: 37$ & -20.85 & 89.43 & 833.77 & Indian Ocean \\
13553 & $4 / 28 / 94$ & $3: 39: 49$ & $23: 14: 47$ & -9.93 & -66.26 & 833.80 & Lima, Peru \\
13591 & $5 / 5 / 94$ & $3: 19: 49$ & $20: 41: 54$ & 29.23 & -99.48 & 834.28 & San Antonio \\
13571 & $5 / 1 / 94$ & $3: 37: 49$ & $21: 27: 06$ & 32.56 & -92.68 & 831.56 & New Orleans \\
13577 & $5 / 2 / 94$ & $3: 07: 49$ & $21: 13: 56$ & 32.75 & -88.47 & 831.92 & New Orleans \\
13568 & $4 / 24 / 94$ & $15: 26: 41$ & $9: 59: 05$ & 39.61 & -81.90 & 763.97 & Cincinnati \\
13574 & $4 / 30 / 94$ & $15: 57: 32$ & $9: 13: 32$ & 44.00 & -101.00 & 765.00 & South Dakota \\
\hline
\end{tabular}

As before we start with the quiet areas, shown separately in Figure 7. The two southernmost records, 13559 and 13565, show very similar behavior to that seen in the Antarctic record measured in band A, file 13082. The amplitude of the signal is essentially flat, with no evidence of any effect from the satellite rotation. The amplitudes of the signals are approximately three times that of the narrower 
band data, which is about the ratio of the bandwidths. This is consistent with an interpretation of there being no significant transmitters in the field of view, just a relatively flat thermal-noise background. Record 12029 was taken at a similar latitude but has a rather different character. It appears to consist of a fairly featureless background, consistent with the first two records, with two distinct bursts of RF power superimposed. The spacing between the bursts is approximately 45 seconds, which is the satellite rotation period, but we suspect this is coincidental. We would expect a regular sinusoidal variation of the signal if it were due to an antenna orientation effect. The sharp-edged nature of these bursts makes us think the intermittent nature of the signal is inherent in the source.

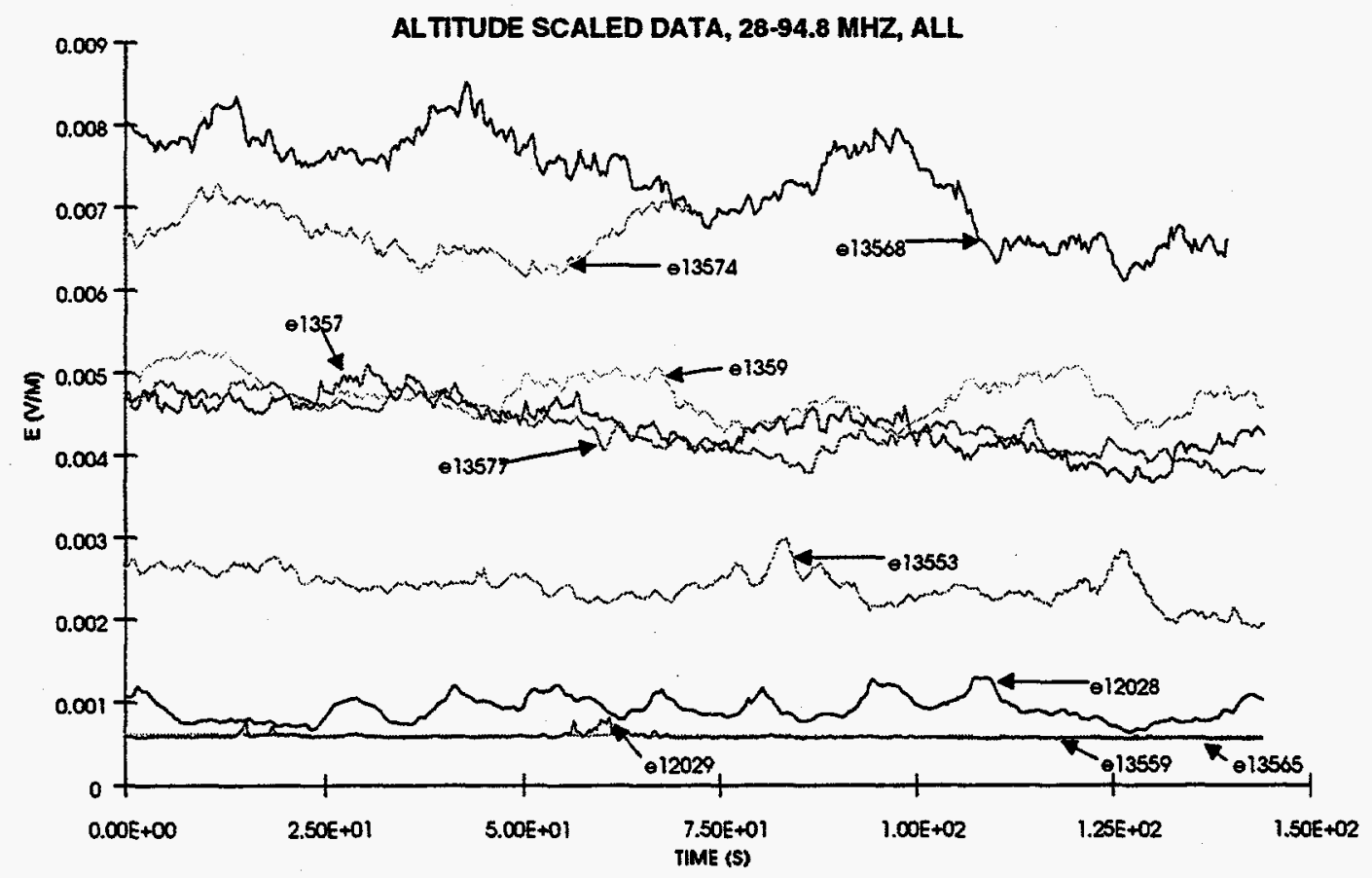

Figure 6

The remaining quiet area record is quite a bit farther north, but the subsatellite point is in the middle of the Indian Ocean and there are no continental land masses in view. This record shows a much larger signal than the more southern ones, with regular variations. This is most likely coming from transmitters on Diego Garcia, which is just on the horizon for this case. A Fourier analysis of the record shows pronounced peaks at 0.022 and $0.066 \mathrm{~Hz}$. The lowerfrequency peak is the usual indicator of a rotation-dependent signal coming from the variation in the satellite antenna pattern. The higher-frequency peak may be a harmonic of this or it may be an inherent feature of the source.

Next we turn to the noisy areas. We have three records taken over the southern United States, 13571, 13577, and 13591, and two records taken at higher latitudes. The data are shown in Figure 8. The three southern U.S. records are remarkably consistent. Records 13571 and 13577 exhibit some variability but do not show strong systematic fluctuations, while the spectrum of record 13591 does 
have a peak at $0.022 \mathrm{~Hz}$. We interpret this to mean that there are a number of more-or-less-equal power noise sources for the first two records, but the third has at least one dominant noise source. The higher-latitude records, 13568 and 13574, are consistent with each other and do not show any pronounced peaks in their spectra.

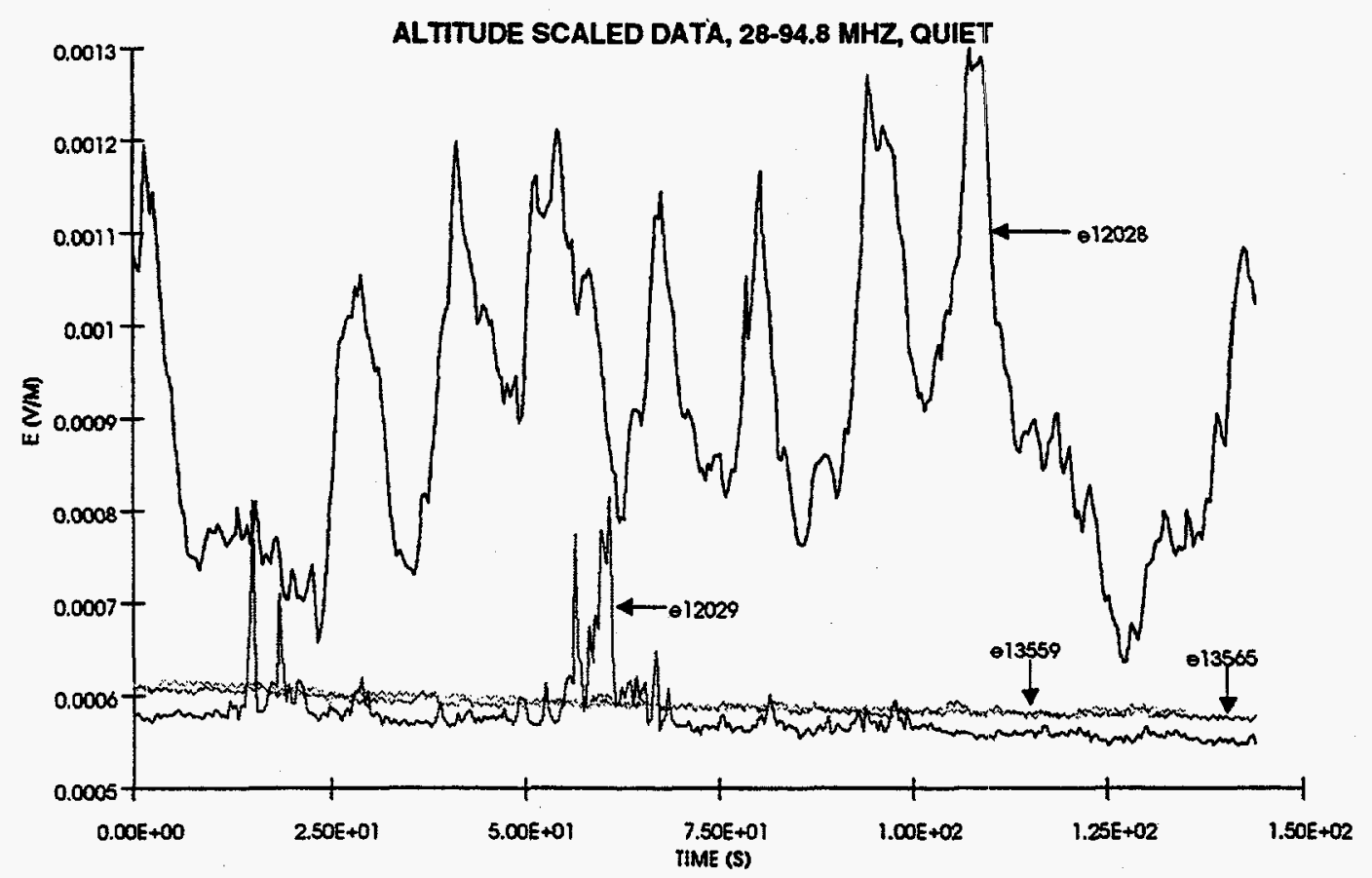

Figure 7

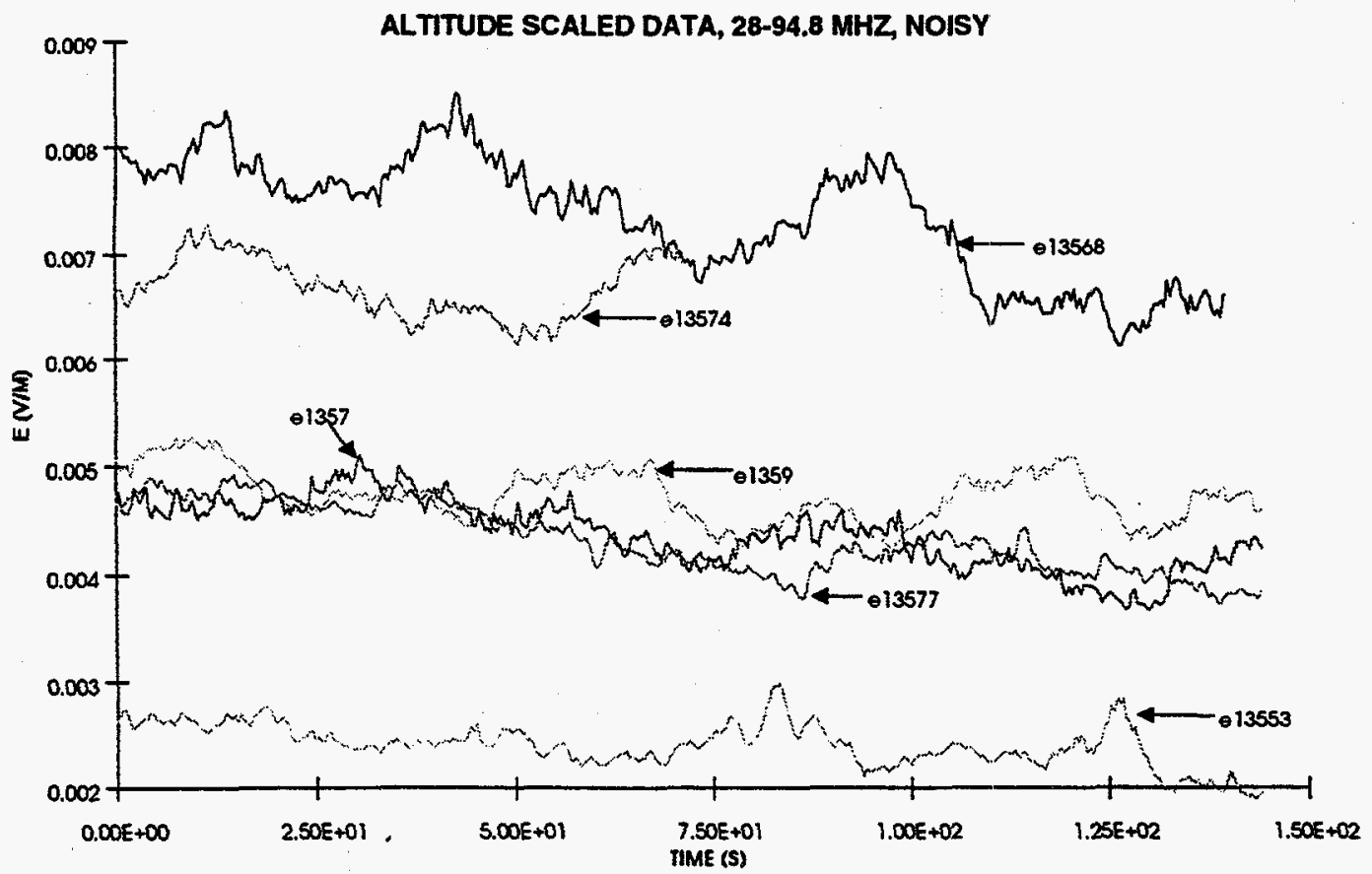

Figure 8 
The last record in this band, 13553, was taken over Peru. It is intermediate in amplitude between the U.S. data and the Antarctic data and shows little amplitude variation. Spectral analysis shows that the variation present has a pronounced peak at $0.022 \mathrm{~Hz}$, indicating a spacecraft-rotation effect.

\section{Band C : 132.3-152.2 MHz}

Next we look at Blackbeard's high band. We begin with a 20-MHz-wide channel spanning 132.3-152.2 $\mathrm{MHz}$. This band does not include continuous transmitters such as television or FM radio, but is used heavily by intermittent sources such as push-to-talk radios and aircraft communications. We therefore expected a highly variable environment. A total of ten records were taken, two over quiet areas and the remaining eight over noisy areas. Details of the records are given in Table 4 and the full set of data is shown in Figure 9. The quiet and noisy records are not as well separated as they were in the low-band data but can be distinguished by the degree of variability of the signals.

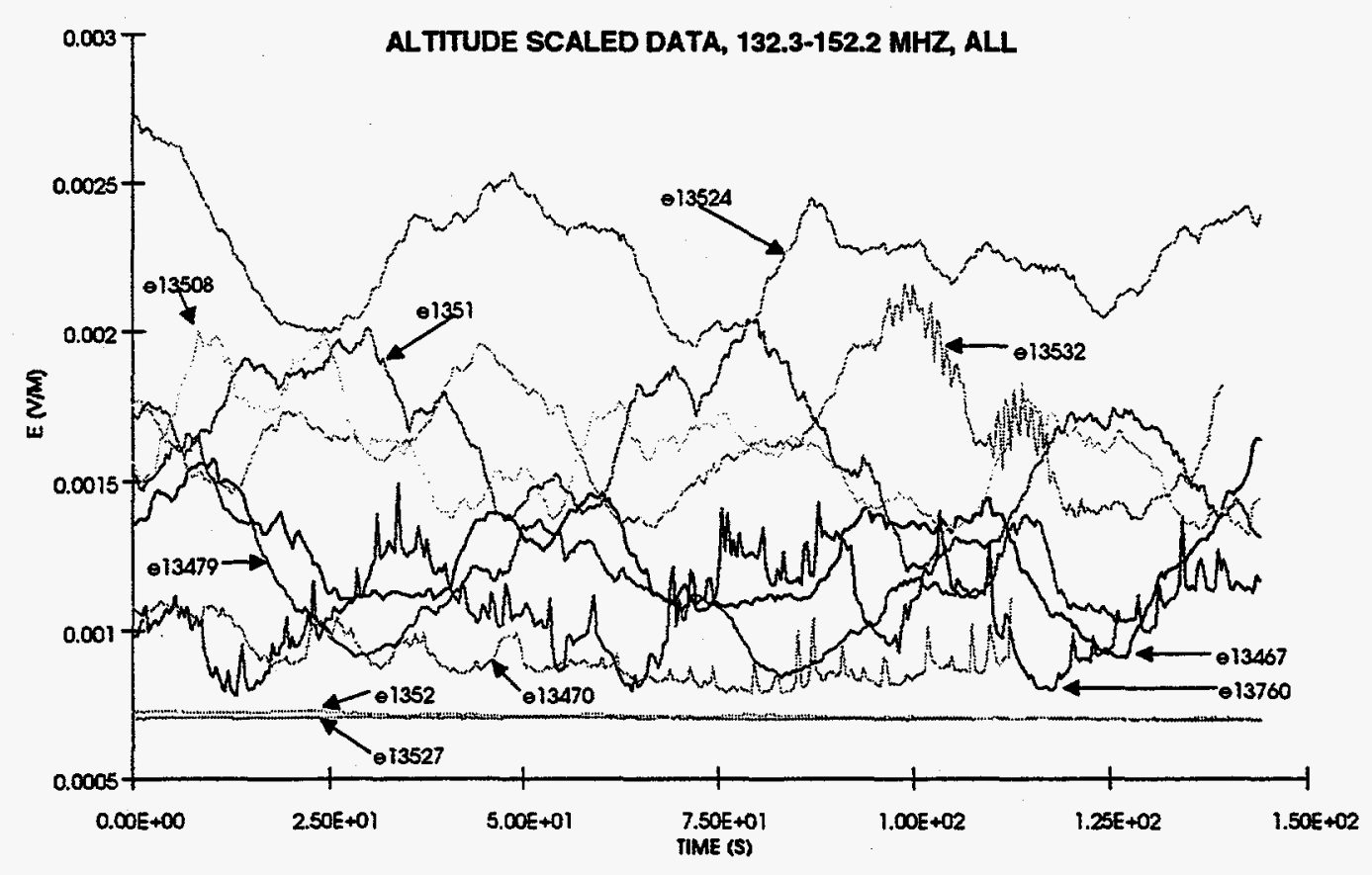

Figure 9

Records 13521 and 13527 were taken at very low latitudes, near Antarctica. They are shown in Figure 10. As was the case with the low-band data from this region, the data show very little variation and do not have any systematic features, which indicates that the satellite was seeing noise whose source is independent of orientation. Curiously, the noise amplitude is about three times higher than seen over this area in band A, although the bandwidth is the same. 
Table 4. 132.3-152.2 MHz Data

\begin{tabular}{lrrrrrrl}
\hline File & \multicolumn{1}{c}{ Date } & \multicolumn{1}{c}{ Time(UT) } & Time (loc) & \multicolumn{1}{c}{ Lat. } & Long. & Alt. (km) & Subsatellite Point \\
\hline 13527 & $4 / 22 / 94$ & $5: 19: 49$ & $5: 24: 10$ & -69.41 & 1.09 & 796.24 & Antarctica \\
13521 & $4 / 21 / 94$ & $5: 44: 49$ & $3: 10: 20$ & -60.39 & -38.62 & 810.11 & S. Georgia Is. \\
13479 & $4 / 11 / 94$ & $8: 39: 49$ & $2: 16: 35$ & 14.84 & -95.81 & 827.60 & Mexico \\
13467 & $4 / 9 / 94$ & $7: 53: 49$ & $2: 12: 54$ & 31.77 & -85.23 & 816.58 & New Orleans \\
13532 & $4 / 23 / 94$ & $5: 56: 49$ & $23: 11: 20$ & 31.92 & -101.37 & 827.34 & Austin \\
13508 & $4 / 19 / 94$ & $6: 14: 49$ & $23: 56: 03$ & 35.24 & -94.69 & 822.62 & St. Louis \\
13515 & $4 / 20 / 94$ & $5: 44: 49$ & $23: 42: 51$ & 35.44 & -90.49 & 823.21 & Memphis \\
13524 & $4 / 21 / 94$ & $19: 52: 49$ & $13: 26: 27$ & 37.01 & -96.59 & 756.24 & Kansas City \\
13760 & $6 / 7 / 94$ & $13: 38: 49$ & $12: 52: 20$ & 43.58 & -11.62 & 835.82 & Lisbon \\
13470 & $4 / 3 / 94$ & $21: 03: 41$ & $17: 29: 43$ & 67.63 & -53.49 & 769.88 & Greenland \\
\hline
\end{tabular}

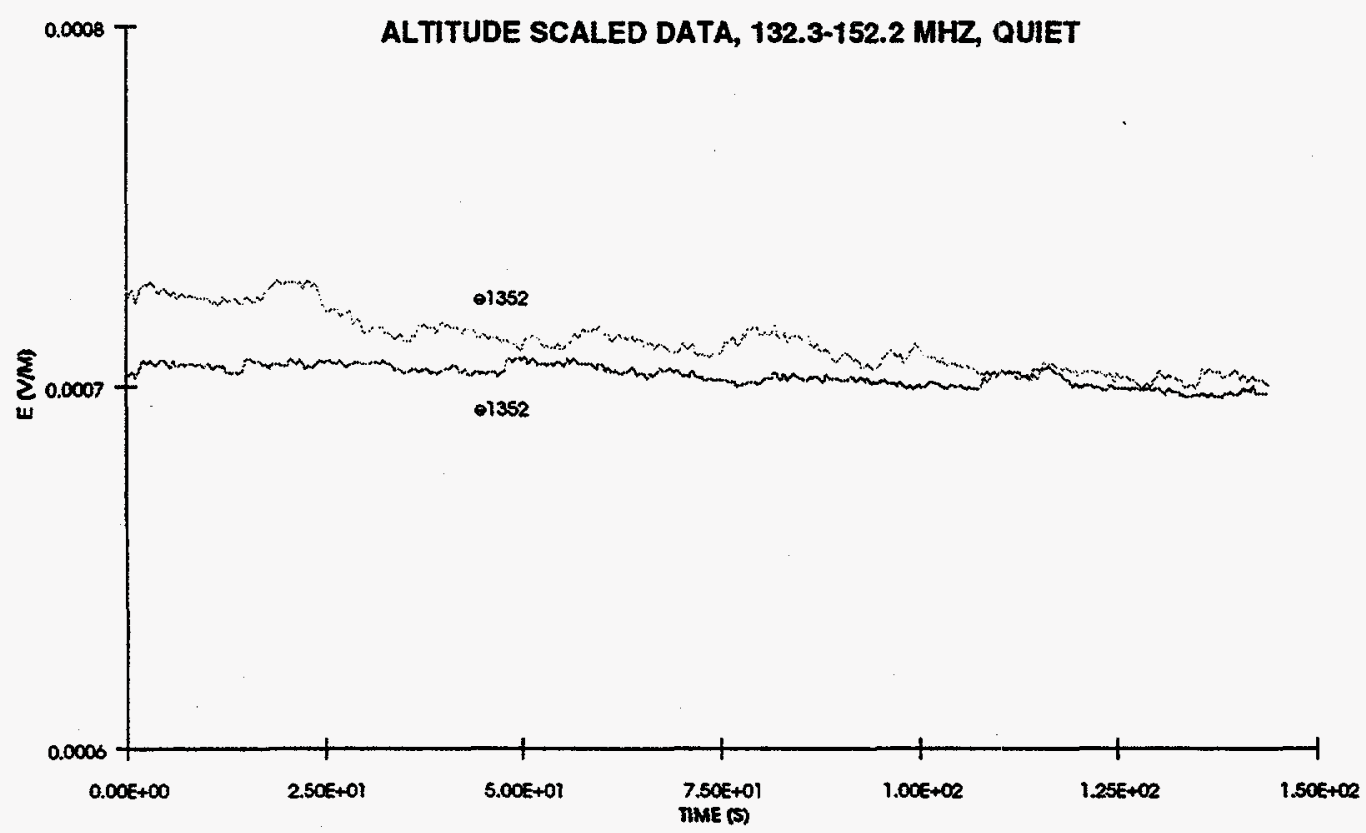

Figure 10

The data from noisy areas are quite variable and is shown in Figure 11. The average amplitude is only about half that for band $A$, which reflects the absence of continuous transmitters. No subclasses of data are evident despite the fact that the locations of the subsatellite points vary widely. The spectra of all of the records except 13470 display clear satellite-rotation effects. 


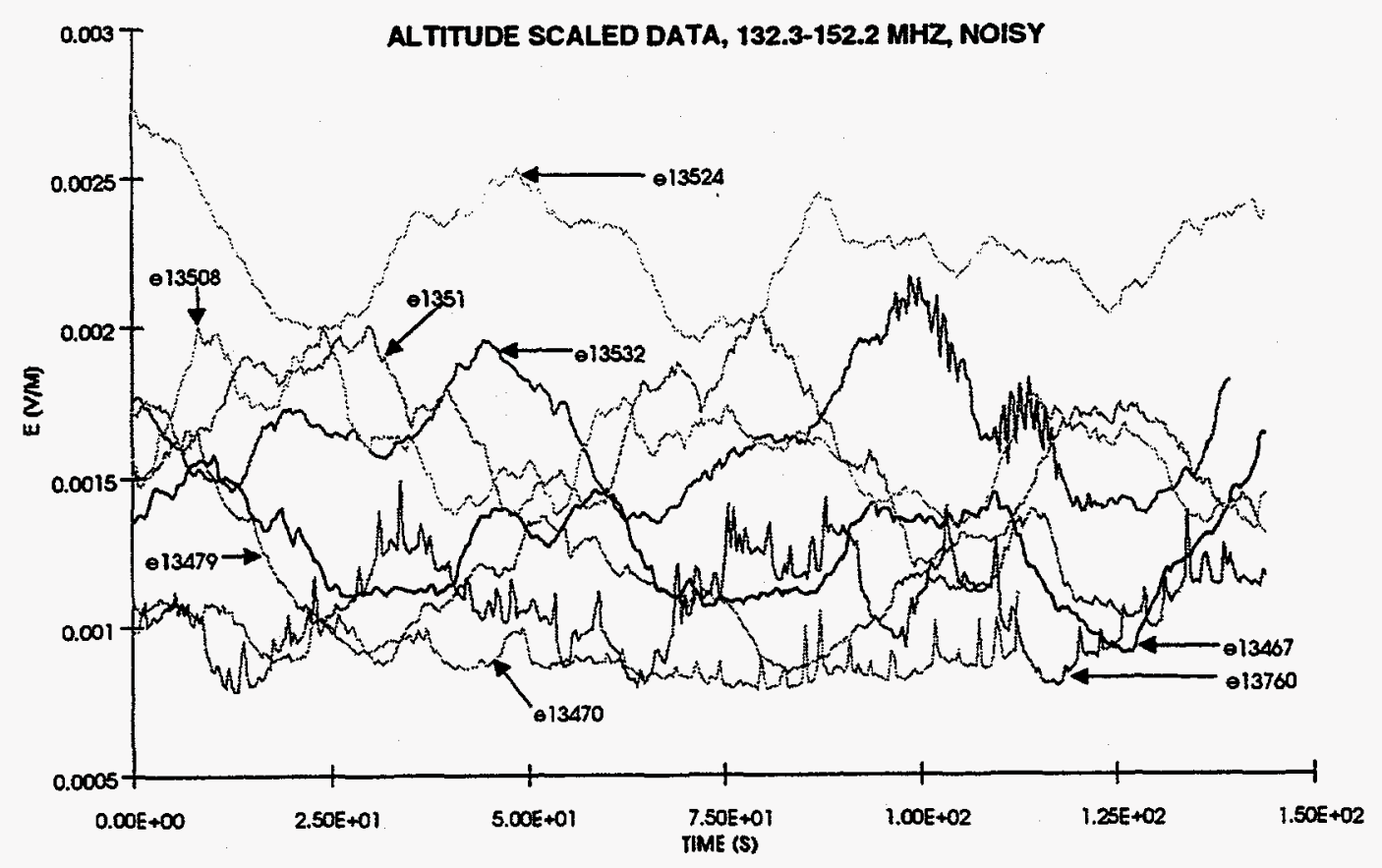

Figure 11

\section{Band D : 107.7-166.0 MHz}

Finally we look at the high band at maximum bandwidth, 107.7-166.0 MHz. This includes a few MHz of the FM band in the United States but is below the upperVHF television channels. It includes airport beacons and some radars as well as the intermittent communications channels discussed previously in band $\mathrm{C}$. We have a total of twelve records, six taken over quiet areas and six over noisy areas. The complete set of data is shown in Figure 12, and details of the times and

Table 5. $107.7-166.0 \mathrm{MHz}$ Data

\begin{tabular}{lrrrrrrl}
\hline File & \multicolumn{1}{c}{ Date } & Time(UT) & Time (loc) & \multicolumn{1}{c}{ Lat. } & \multicolumn{1}{c}{ Long. } & Alt. (km) & Subsatellite Point \\
\hline 13616 & $5 / 10 / 94$ & $3: 09: 49$ & $5: 13: 01$ & -63.73 & 30.80 & 762.57 & Antarctica \\
13619 & $5 / 11 / 94$ & $0: 59: 49$ & $5: 16: 56$ & -62.17 & 64.28 & 760.41 & Antarctica \\
13458 & $4 / 1 / 94$ & $22: 44: 41$ & $3: 02: 34$ & -42.52 & 64.47 & 830.77 & Indian Ocean \\
13455 & $4 / 7 / 94$ & $9: 04: 49$ & $3: 37: 56$ & -4.96 & -81.72 & 832.59 & Peru \\
13461 & $4 / 8 / 94$ & $8: 26: 00$ & $14: 11: 12$ & -24.50 & 86.30 & 820.00 & Indian Ocean \\
13594 & $5 / 6 / 94$ & $2: 48: 49$ & $20: 22: 06$ & 32.69 & -96.68 & 833.78 & Dallas \\
13599 & $5 / 7 / 94$ & $2: 18: 49$ & $20: 08: 56$ & 32.89 & -92.47 & 834.05 & Dallas \\
13611 & $5 / 9 / 94$ & $1: 18: 49$ & $19: 42: 37$ & 33.28 & -84.05 & 834.54 & Atlanta \\
13602 & $5 / 7 / 94$ & $16: 56: 49$ & $10: 07: 32$ & 40.16 & -102.32 & 770.28 & Denver \\
13605 & $5 / 8 / 94$ & $1: 45: 49$ & $19: 32: 18$ & 42.62 & -93.38 & 831.26 & Omaha \\
13608 & $5 / 8 / 94$ & $16: 27: 49$ & $10: 03: 08$ & 43.18 & -96.17 & 773.60 & Omaha \\
13464 & $4 / 8 / 94$ & $23: 12: 00$ & $2: 56: 14$ & 67.91 & 56.06 & 759.00 & Novya Zemlya \\
\hline
\end{tabular}




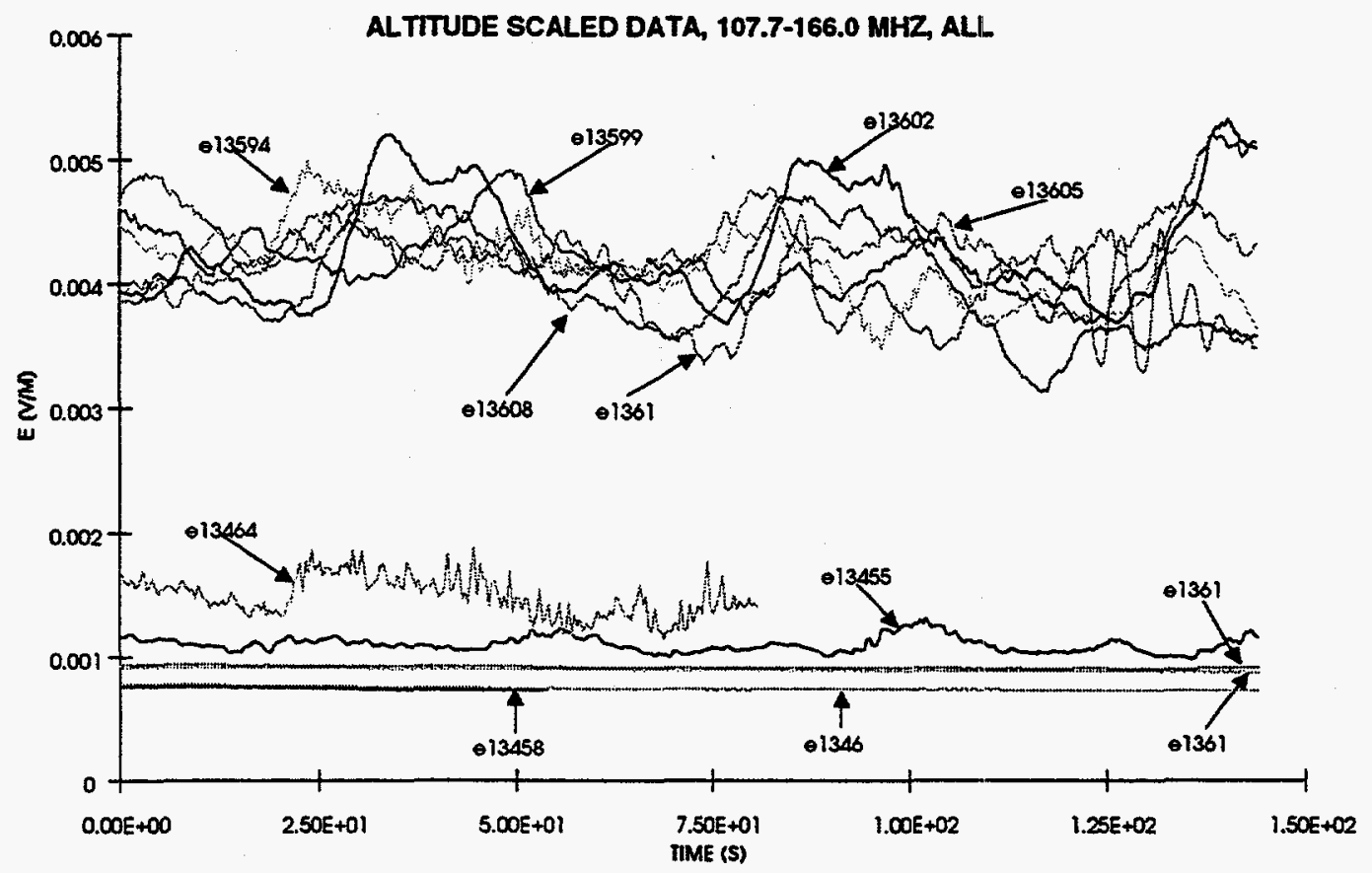

Figure 12

subsatellite points are given in Table 5. Once again, the data in this band fall into two clearly separated groups.

As usual, we start with the quiet area data, shown in Figure 13. There are two records taken over Antarctica, 13616 and 13619. As was the case in the other bands, the amplitudes of the signals are quite constant and do not shown any satellite-orientation effects. Interestingly, the signal strength is only about $30 \%$ higher than for band $\mathrm{C}$ despite the fact that the bandwidth is almost three times larger. This clearly shows that the noise spectrum is not "white" in band D. Even more intriguing are the records taken over the southern Indian Ocean, 13461 and 13458. Unlike the Indian Ocean records in the low band these do not have Diego Garcia in the field of view, and now we see that they are also very smooth and of even lower amplitude than the Antarctic records. This may be a satelliteorientation effect. Record 13455, taken just off the coast of Peru, shows considerable variation and a strong periodic behavior at periods of 45 and 22.5 seconds, which indicates a small number of localized sources in view. The final record in this set is 13464 , which was taken at a very high northern latitude, over the Russian island of Novya Zemlya. This record shows a dramatic, rapid variation unlike most of the other data. Similar effects were seen in other bands in data taken over the Indian Ocean and in view of Diego Garcia; perhaps this is due to a radar or other rapidly modulated source. The record is too short to clearly establish whether there are spacecraft-rotation effects present. 


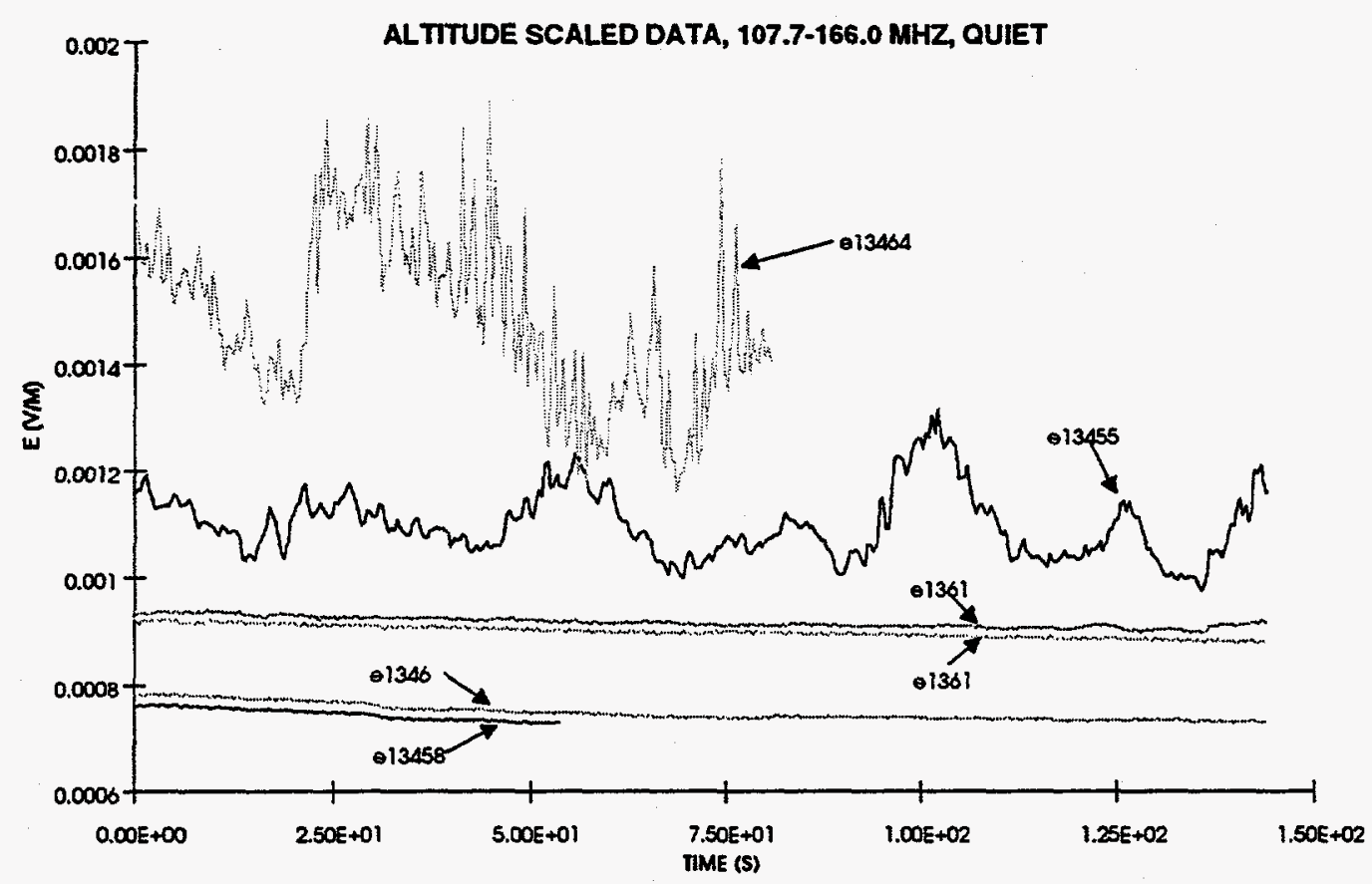

Figure 13

The noisy area data in this band, shown in Figure 14, was taken over the United States. The data are variable but on average consistent, with an amplitude approximately the same as seen in band B. Records 13602,13605, and 13608 exhibit a strong $0.022 \mathrm{~Hz}$ variation while records 13594, 13599, and 13611 do not.

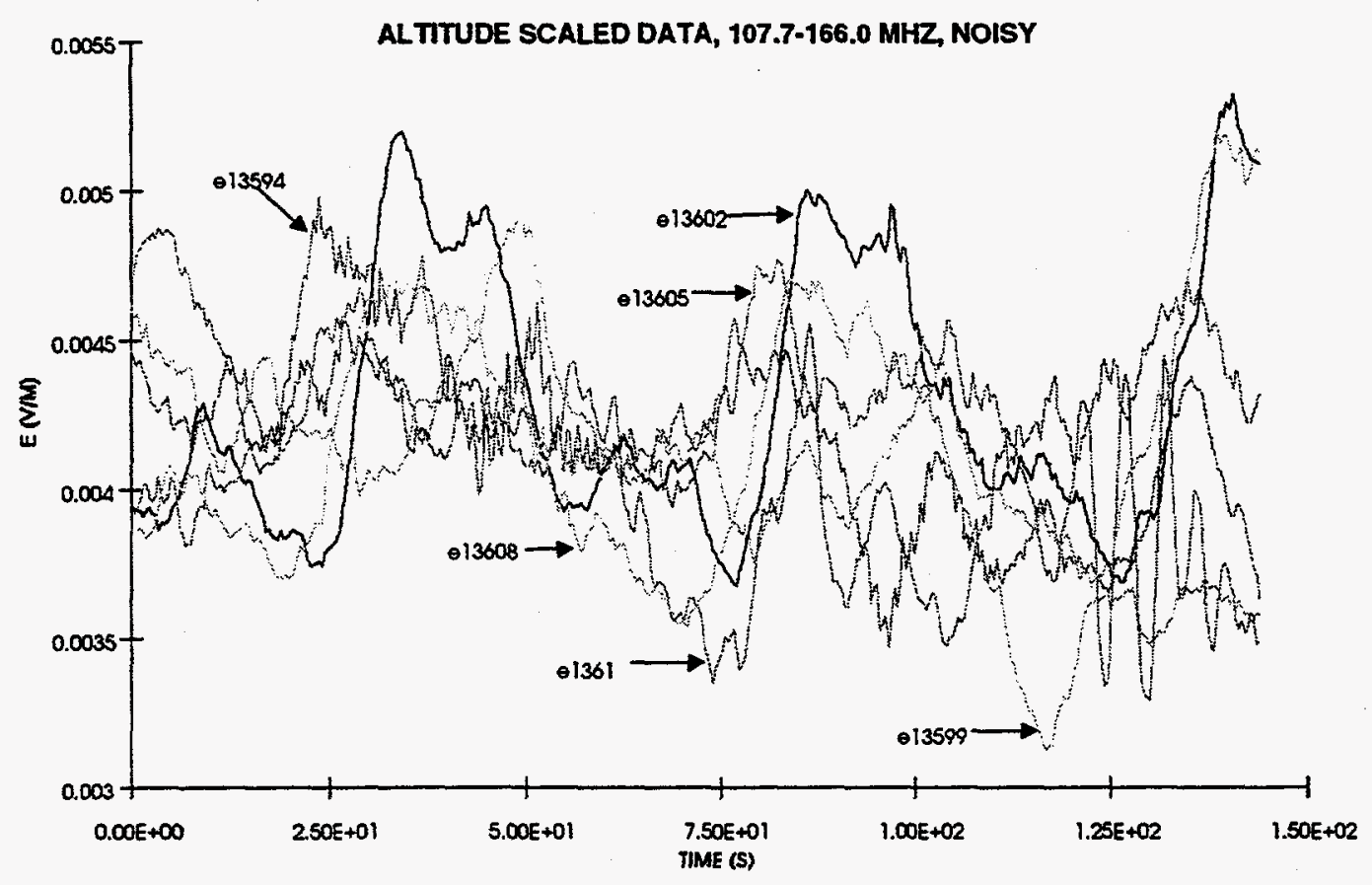

Figure 14 


\section{References :}

1. D.N. Holden, C.P. Munson, and J.C. Devenport, "Satellite Observations of Transionospheric Pulse Pairs," Geophysics Research Letters, 22(8), 889-892 (1995).

2. R.S. Massey and D.N. Holden, "Phenomenology of Transionospheric Pulse Pairs," Radio Science, 30(5), 1645-1659 (1995). 